\title{
Large-scale transcriptome comparison reveals distinct gene activations in wheat responding to stripe rust and powdery mildew
}

Hong Zhang ${ }^{\dagger}$, Yongzheng Yang ${ }^{\dagger}$, Changyou Wang, Min Liu, Hao Li, Ying Fu, Yajuan Wang, Yingbin Nie, Xinlun Liu and Wanquan Ji*

\begin{abstract}
Background: Stripe rust (Puccinia striiformis f. sp. tritici; Pst) and powdery mildew (Blumeria graminis f. sp. tritici; Bgt) are important diseases of wheat (Triticum aestivum) worldwide. Similar mechanisms and gene transcripts are assumed to be involved in the host defense response because both pathogens are biotrophic fungi. The main objective of our study was to identify co-regulated mRNAs that show a change in expression pattern after inoculation with Pst or Bgt, and to identify mRNAs specific to the fungal stress response.

Results: The transcriptome of the hexaploid wheat line N9134 inoculated with the Chinese Pst race CYR 31 was compared with that of the same line inoculated with Bgt race E09 at 1,2, and 3 days post-inoculation. Infection by Pst and Bgt affected transcription of $23.8 \%$ of all T. aestivum genes. Infection by Bgt triggered a more robust alteration in gene expression in $\mathrm{N} 9134$ compared with the response to Pst infection. An array of overlapping gene clusters with distinctive expression patterns provided insight into the regulatory differences in the responses to Bgt and Pst infection. The differentially expressed genes were grouped into seven enriched Kyoto Encyclopedia of Genes and Genomes pathways in Bgt-infected leaves and four pathways in Pst-infected leaves, while only two pathways overlapped. In the plant-pathogen interaction pathway, N9134 activated a higher number of genes and pathways in response to Bgt infection than in response to Pst invasion. Genomic analysis revealed that the wheat genome shared some microbial genetic fragments, which were specifically induced in response to Bgt and Pst infection.
\end{abstract}

Conclusions: Taken together, our findings indicate that the responses of wheat N9134 to infection by Bgt and Pst shows differences in the pathways and genes activated. The mass sequence data for wheat-fungus interaction generated in this study provides a powerful platform for future functional and molecular research on wheat-fungus interactions.

Keywords: Bread wheat, Stripe rust, Powdery mildew, RNA-Seq, Gene expression

\section{Background}

Plants are constantly exposed to microbes in natural and agricultural ecosystems. To be pathogenic, most microbes must access the plant's internal tissues, either by penetrating the plant surface directly or by entering through wounds or natural openings such as stomata $[1,2]$. Furthermore, the pathogen must pierce through the cell wall, and often the host plasma membrane, to

\footnotetext{
*Correspondence: jiwanquan2008@126.com

${ }^{\dagger}$ Equal contributors

State Key Laboratory of Crop Stress Biology for Arid Areas, College of Agronomy (Northwest A\&F University), Yangling, Shaanxi 712100, China
}

be infective. Plants wield typical basal and resistance (R) gene-mediated defense mechanisms and mount a defensive response to pathogen attack to delay or arrest potential pathogenic microorganism growth [3] through pathogen-associated molecular pattern-triggered immunity (PTI) and effector-triggered immunity (ETI) systems. Current analysis of plant immunity has moved towards an integrated view of plant-pathogen interactions [4]. Using oligonucleotides, cDNA microarrays and transcriptome analysis, many comprehensive analyses of stress-induced changes in gene expression in model plants with small genomes have been undertaken, and consequently many 
genes associated with pathogenic stress responses in plants are known. However, numerous fundamental molecular aspects remain unknown, such as the unique and common signaling components of PTI and ETI, and the induced host components that effect resistance. A global gene expression approach is useful for elucidating the molecular mechanisms of wheat-fungus interactions, particularly the application of next-generation sequencing to study important non-model host-pathogen systems, such as wheat rusts [5].

Stripe rust (Puccinia striiformis f. sp. tritici; Pst) and powdery mildew (Blumeria graminis f. sp. tritici; Bgt) are important fungal diseases of wheat (Triticum aestivum) in many wheat-growing regions of the world. The filamentous powdery mildew and rust fungi develop haustoria within the lumen of the host cell, which function to absorb nutrients. A number of studies on wheat-rust interactions have been carried out using the Affymetrix ${ }^{\bullet}$ GeneChip $^{\odot}$ Wheat Genome Array (Affymetrix, Santa Clara, CA, USA) [6,7] and cDNA-AFLP analysis [8]. Powdery mildew infection has been studied in grapevine [9], Hordeum [10] and Arabidopsis [11] with the Affymetrix GeneChip Wheat Genome Array, cDNA-AFLP, and cDNA microarrays, respectively. However, a complete gene expression profile for the response to the stripe rust and powdery mildew pathogens in the same wheat germplasm is still lacking. Compared with other plants with smaller genomes, the use of most genetic and molecular techniques to study genes involved in wheat-Pst or wheat-Bgt interactions has been limited, because hexaploid wheat has a large and complex genome and its transformation is difficult, and both fungi show sexual reproduction and irreversible deletion of genes dispensable for biotrophy $[10,12]$. Use of the Affymetrix GeneChip Wheat Genome Array is often restricted by the known gene sequences arrayed on the chip, with a limited number of expressed sequence tags (ESTs) non-specific to different wheat materials, whereas cDNAAFLP is universally applicable for any organism or interaction without prior sequence information required, although false positives may frequently be observed because of technical reasons. In contrast, RNA sequencing (RNA-Seq) is not dependent on pre-existing databases of expressed genes and, therefore, provides an unbiased view of gene expression profiles. In the present study, using Illumina deep sequencing, we undertook a transcriptome analysis of leaves from different plants of the same wheat line, N9134, subjected to both Pst and Bgt stress treatments. The main objective was to identify co-regulated mRNAs that show a change in expression pattern after inoculation with Pst or Bgt, and to identify mRNAs specific to the fungal stress response. This is the first study to compare two biotic stresses using a global expression profiling strategy in the same wheat genetic background. Through a comparison of different pathogen stress treatments with biological replicates, we reasoned that we could better identify both shared and stimulus-specific responses.

\section{Results}

In the present study, cDNA libraries were constructed from leaves inoculated with Pst or Bgt at 0, 1, 2 and 3 days postinoculation (dpi) with three biological replicates, and then sequenced using the Illumina $\mathrm{HiSeq}^{\mathrm{Tm}} 2000$ platform. After cleaning and checking the read quality, we obtained almost 46.75 million $101 \mathrm{bp}$ paired-end clean reads. Among the clean reads, 100\% had quality scores at the Cycle Q20 level (a base quality greater than 20 and an error probability of 0.01). The data sets were deposited in the NCBI Sequence Read Archive (accession number PRJNA243835). Because of deficiencies in the reference genome sequence, these reads were de novo assembled using the Trinity platform software, resulting in 186,632 unigenes with N50 length of $743 \mathrm{bp}$, of which 89,672 unigenes were annotated after Blast searches of the GenBank Nr, SwissProt, KEGG, COG and GO databases. The length of 22,825 unigenes was more than $1 \mathrm{~kb}$ and contained 4,837 simple sequence repeat sites.

As an aid to examining gene expression level distributions, the reads per kilobase of exon model per million of aligned reads (RPKM) values were calculated as normalized expression estimates for each gene model in each sample. Also, correlation coefficients were calculated based on the log-transformed RPKM values after eliminating genes with a zero count in any of the three replicates. The correlation coefficient values ranged from 0.930 to 0.994 (Additional file 1: Table S1), indicating there was a strong correlation between replicates. A generalized linear model was applied based on a negative binomial distribution and an overall test was conducted to determine which genes varied in expression among any of the seven treatment groups, where a treatment group was defined by a strain-by-induction condition combination (see Methods for details). Setting fold change $\geq 2$ and the false discovery rate (FDR) at $1.0 \%$ using the method of Benjamini and Hochberg [13], statistical analysis with DESeq identified 10,583 genes as differentially expressed among the six treatment groups compared with non-inoculated leaves as the control. Of these genes, the length of 7,298 genes exceeded $1 \mathrm{~kb}$. Table 1 lists details of the differentially expressed genes (DEGs) and annotation numbers that were detected at the different time points in response to the fungal stress treatments.

To evaluate the reliability of our RNA-Seq and de novo assembled results, quantitative real-time PCR (qPCR) was performed on eight selected genes of interest using RNA samples as a fourth replication. These genes were selected to represent a wide range of expression levels and patterns under fungal infection. Six gene expression patterns in response to Bgt stress showed strong agreement and were highly correlated in the RNA-Seq and 
Table 1 Statistical table of differentially expressed genes number and annotated DEGs

\begin{tabular}{|c|c|c|c|c|c|c|}
\hline Type & S1_vs_Ck & S2_vs_Ck & S3_vs_Ck & P1_vs_Ck & P2_vs_Ck & P3_vs_Ck \\
\hline num & 2,208 & 991 & 1,065 & 4,549 & 5,275 & 5,525 \\
\hline up & 900 & 324 & 326 & 2,771 & 1,548 & 1,718 \\
\hline down & 1,308 & 667 & 739 & 1,778 & 3,727 & 3,807 \\
\hline $\mathrm{nr}$ & 2,088 & 913 & 1,011 & 4,213 & 4,789 & 4,882 \\
\hline SwissProt & 1,811 & 765 & 869 & 3,700 & 4,058 & 4,029 \\
\hline GO & 1,871 & 783 & 894 & 3,786 & 4,181 & 4,213 \\
\hline KEGG & 432 & 168 & 228 & 888 & 868 & 899 \\
\hline COG & 853 & 301 & 375 & 1,685 & 1,696 & 1,660 \\
\hline
\end{tabular}

Note: $\mathrm{P}$ represents powdery mildew E09 inoculation condition; $\mathrm{S}$ represents stripe rust pathogen CYR 31 inoculation; Ck represents the samples without pathogen stress as control. The number of 1, 2 and 3: N9134 infected at 1, 2 and 3 dpi, respectively.

qPCR analyses (Additional file 1: Figure S1). Additionally, six previously studied full-length genes were aligned with the present assembled unigene database and showed identities of up to $99.6 \%$.

\section{More robust response to Bgt- than Pst-induced stress in bread wheat}

Although transcript levels do not necessarily reflect the amount of final active protein product, for simplicity, increased transcript abundance is often referred to as induced gene expression. Genes that were differentially expressed between non-inoculated and inoculated leaves were determined with RNA-Seq. Cluster analysis showed that the expression profiles of DEGs varied significantly in response to Bgt and Pst in the N9134 genotype (Figure 1).

For the Bgt test, using inoculated leaf samples, expression of 9,114 DEGs was detected, of which 6,292 genes exceeded $1 \mathrm{~kb}$. As shown in Table 1 and (Additional file 1: Figure S2), comparison of inoculated and non-inoculated leaves showed that 4,549, 5,275 and 5,525 genes were differentially expressed at 1,2 , and $3 \mathrm{dpi}$, respectively. Of these genes, 2,771 and 1,778 were up- and down-regulated at $1 \mathrm{dpi}, 1,548$ and 3,727 at $2 \mathrm{dpi}$, and 1,718 and 3,807 at 3 dpi, respectively. In the Pst CYR 31 inoculation treatment, 3,359 DEGs showed significantly lower expression than that in the Bgt E09 stress treatment. Of these genes, 2,208 (900 up- and 1,308 down-regulated), 991 (324 up- and 667 down-regulated), and 1,065 (326 up- and 739 downregulated) were differentially expressed at 1, 2, and $3 \mathrm{dpi}$, respectively. The number of up-regulated genes at $1 \mathrm{dpi}$ was almost double those at 2 and $3 \mathrm{dpi}$ after infection by $B g t$, whereas the number at $1 \mathrm{dpi}$ was almost three-times higher than those at 2 and $3 \mathrm{dpi}$ after Pst infection. This substantiated the contention that $1 \mathrm{dpi}$ is the most important time point for wheat to respond to fungal attack by expression of race-specific resistance genes.

To further test the variation in response to Pst and Bgt inoculation, we compared gene expression in response to Pst CYR 31 versus Bgt E09 inoculation with fold change $\geq 4$ and FDR at $1.0 \%$. Additional file 1: Figure S3 shows that 8,110 genes were differentially expressed in response to Bgt infection in comparison with Pst infection, of which 2,208 and 804 genes were up- and downregulated at $1 \mathrm{dpi}, 1,855$ and 2,456 at $2 \mathrm{dpi}$, and 1,725 and 1,973 at $3 \mathrm{dpi}$, respectively. This result indicated that the wheat resistance response to $B g t$ inoculation triggered more robust alteration in gene expression than that observed in response to Pst inoculation.

\section{Gene characterization of stimulus-specific responses to Pst or Bgt}

To facilitate a closer comparison of Pst-induced genes with Bgt-induced genes, a pairwise comparison was conducted after DEGs were annotated using KEGG classifications and GO assignments. All resistance-specific genes were analyzed using MAS to identify the metabolic pathways in which they function. The GO enrichment results are shown in Additional file 1: Tables S2 (biological process), S3 (molecular function), and S4 (cellular component). When the FDR-corrected $P$-value was set at 0.05 , seven significant enriched KEGG pathways for Bgt-induced stress and four for Pst-induced stress versus the non-stressed control were identified. Two KEGG pathways overlapped between the two infection treatments (Table 2), namely ubiquinone and other terpenoid-quinone biosynthesis and photosynthesis-antenna proteins. As there were insufficient pathway annotations in KEGG, the pathways were retrieved using the DEGs comparing Pst infection versus Bgt infection directly at the specific time points and are listed in Additional file 1: Table S5. Taken together, 16 KEGG pathways were identified.

Phenylalanine metabolism, phenylpropanoid biosynthesis, alpha-linolenic acid metabolism, flavonoid biosynthesis, and phenylalanine, tyrosine and tryptophan biosynthesis showed specific significant differential enrichment in wheat in response to Bgt-induced stress, whereas carotenoid biosynthesis and carbon fixation in photosynthetic organisms showed the opposite differential performance in response to Pst-induced stress (Table 2). The analysis of KEGG pathways showed that 11 specific pathways responded to $B g t$ 


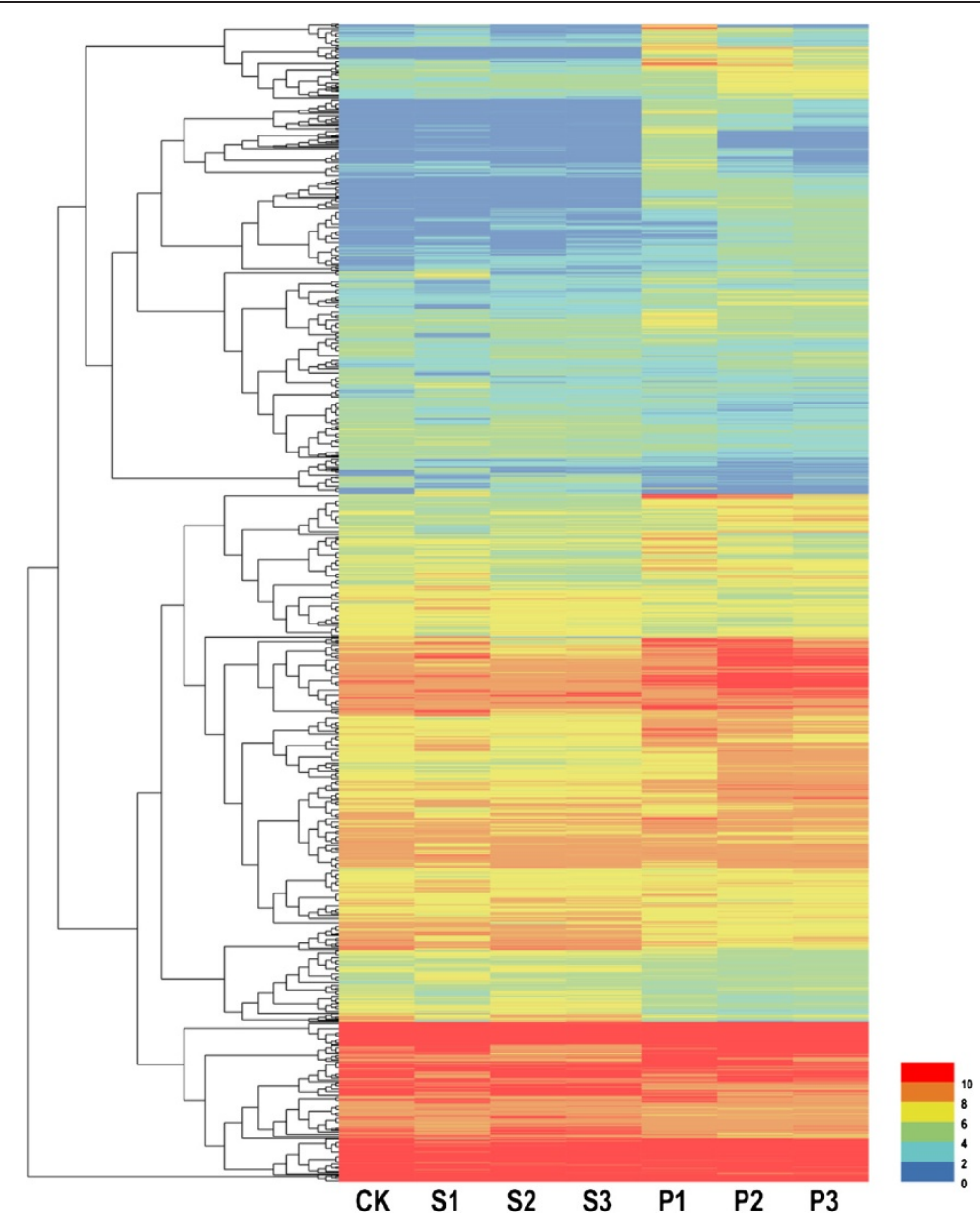

Figure 1 Hierarchical clustering of differentially-expressed genes (DEGs) in resistant bread wheat accession N9134 to Bgt and Pst infection. The signal ratios are shown in a red-green color scale, where red represents up-regulation and green represents down-regulation. Each column (Ck, S1, S2, S3, P1, P2 and P3) represents the mean expression value of the RNA-Seq obtained from three biological replicates and each row represents a differentially-expressed genes. Ck: resistant wheat N9134 exempt from pathogen stress as control; S1, S2 and S3: N9134 infected with stripe rust pathogen CYR 31 at 1, 2 and 3 dpi, respectively; P1, P2 and P3: N9134 infected with powdery mildew pathogen E09 at 1, 2 and 3 dpi, respectively.

infection and four specific pathways responded to Pst infection, although the $P$ values were not significant for those present in the KEGG database (Additional file 1: Table S6). Under Pst-induced stress, no DEGs were detected in the mismatch repair, non-homologous end-joining, nucleotide excision repair, DNA replication, and RNA polymerase pathways, which are all involved in genetic information processing. Moreover, ABCB1 (K05658) in ABC transporters, mitochondrial trans-2-enoyl-CoA reductase (K07512) in fatty acid elongation, lipoyl (octanoyl) transferase (K03801) in lipoic acid metabolism, biotin synthase (K01012) in biotin metabolism, glutamate decarboxylase, gamma-glutamyl transpeptidase and cysteamine dioxygenase in taurine and hypotaurine metabolism, and N-glycan biosynthesis were specifically enriched in response to Bgt inoculation. The number of dysregulation enzymes reaches to eight in $\mathrm{N}$ glycan biosynthesis especially. In addition, seven distinct
KEGG pathways were retrieved with MAS3.0 after comparative analysis of genes differentially expressed in response to Pst- and Bgt-induced stress, namely nitrogen metabolism, diterpenoid biosynthesis, stilbenoid, diarylheptanoid and gingerol biosynthesis, valine, leucine and isoleucine degradation, photosynthesis, benzoxazinoid biosynthesis, and ribosome pathway. Genes encoding components of conserved protein complexes, such as the proteasome and ribosome, are coexpressed in other organisms and show very similar expression profiles [14]. Surprisingly, half of the enzymes (70 out of 143) showed dysregulated expression in the ribosome pathway in response to Bgt, whereas 32 dysregulated enzymes were detected in response to Pst-induced stress (Additional file 1: Figure S4). This result indicated that the ribosome pathway is one of the most complex and important pathways regulating genes antagonistic to fungal infection, and that 
Table 2 Significant KEGG pathway of stimulus-specific responses on Pst or Bgt

\begin{tabular}{|c|c|c|c|c|c|c|}
\hline \multirow[t]{2}{*}{ KEGG pathway } & \multicolumn{5}{|c|}{ Correct-p value } & \multirow{2}{*}{$\begin{array}{c}\text { No of } \\
\text { dysregulated } \\
\text { enzymes }\end{array}$} \\
\hline & $\mathbf{P}$ & P only & PS & S only & $\mathbf{S}$ & \\
\hline ko00360 Phenylalanine metabolism & $0.00055^{*}$ & $0.00691^{*}$ & 1 & 1(3.06) & 1 & 10 \\
\hline ko00940 Phenylpropanoid biosynthesis & $0.00559^{*}$ & $0.02167^{*}$ & 1 & 1 & 1 & 30 \\
\hline ko00592 alpha-Linolenic acid metabolism & $0.01487^{*}$ & $0.00516^{*}$ & 1 & 1 & 1 & 13 \\
\hline ko00130 Ubiquinone and other terpenoid-quinone biosynthesis & $0.01643^{*}$ & 1 & $0.01345^{*}$ & 1 & 0.10249 & 7 \\
\hline ko00196 Photosynthesis - antenna proteins & $0.00570^{*}$ & 1 & $1.89 \mathrm{E}-05^{*}$ & 1 & $0.00138^{*}$ & 12 \\
\hline ko00941 Flavonoid biosynthesis & $0.03179^{*}$ & 0.10650 & 1 & 1 & 1 & 25 \\
\hline Ko00400 Phenylalanine, tyrosine and tryptophan biosynthesis & 0.06556 & $0.03537^{*}$ & 1 & 1 & 1 & 19 \\
\hline Ko00710 Carbon fixation in photosynthetic organisms & 1 & 1 & $6.24 \mathrm{E}-05^{*}$ & 1 & $0.00296^{*}$ & 22 \\
\hline Ko00906 Carotenoid biosynthesis & 1 & 1 & 1 & 0.72054 & $0.03038^{*}$ & 20 \\
\hline ko00910 Nitrogen metabolism & 0.16142 & 1 & 1 & - & 1 & 14 \\
\hline ko00904 Diterpenoid biosynthesis & 0.25882 & 0.12611 & 1 & - & 1 & 17 \\
\hline ko00945 Stilbenoid, diarylheptanoid and gingerol biosynthesis & 0.27716 & 0.0556 & 1 & - & $1(2.5)$ & 7 \\
\hline Ko03010 Ribosome & 1 & 1 & $1(4.43)$ & 0.92345 & 1 & 70 \\
\hline ko00280 Valine, leucine and isoleucine degradation & 1 & 1 & 0.761726 & 1 & 1 & 27 \\
\hline ko00195 Photosynthesis & 1 & 1 & 0.465078 & - & 1 & 24 \\
\hline ko00402 Benzoxazinoid biosynthesis & 1 & 1 & 1 & - & 1 & 2 \\
\hline
\end{tabular}

Note: All KEGG pathways were retrieved from MAS3.0 molecule annotation system. Some plant-specific pathways were computed repeat by extracting genes from comparing Pst with Bgt stress. The values of Correct-p were given and those less than 0.05 were marked with asterisks. Some enriched KEGG pathways with the high enrichment factor were noted and the factor was given in brackets. The indistinctive pathway that was listed (bottom 7 lines) had $p$-value $<0.05$ for analysis DEGs from Pst vs Bgt.

further differential internal remodeling in response to different fungal signals is required.

This analysis showed that the nine functional pathways with $P$-values $<0.05$ after FDR correction were significantly associated with resistance to fungal infection, whereas the stimulus-specific responses were more robust and 16 pathways included DEGs in comparison of the Pst and Bgt treatments at 1, 2, and 3 dpi (Additional file 1: Table S5). These results further confirmed the differences in gene regulation and pathways involved in wheat-Pst interaction from those involved in wheat-Bgt interaction.

\section{Identification of DEGs shared by Pst and Bgt infection responses}

From aforementioned information, a considerable number of DEGs would have overlapped between the Bgt and Pst inoculation treatments. Those genes that were significantly regulated in the defense response to both fungi were identified from among the 1,682 overlapping DEGs with annotated information and are listed in Table 3. The overlapping DEGs at each time point are shown in a Venn diagram (Additional file 1: Figure S5). These genes were annotated into $30 \mathrm{GO}$ biological processes with $P$-values $<0.001$ and considerably dysregulated gene numbers. Protein tetramerization, oxidation-reduction process, xenobiotic metabolic process, response to hypoxia, response to abiotic stimulus, starch biosynthetic process, and cholesterol biosynthetic process were not only significantly enriched but also showed a higher number of dysregulated genes than expected $(\mathrm{S} / \mathrm{E}>1.5)$ (Table 3$)$. The number of dysregulated genes in the categories of regulation of $\mathrm{T}$ cell-mediated cytotoxicity, ketone body biosynthetic process, and ergosterol biosynthetic process was doubled that expected ( $\mathrm{S} / \mathrm{E} \mathrm{8,4}$, and 2.4 times, respectively), although there seemed to be a relatively smaller number of DEGs in these three pathways than in the other above-mentioned ones. The GO pedigree analysis indicated that the third main GO hierarchy of biological processes in which the shared genes may participate consisted of response to single-organism stimuli and metabolic process.

Inspired by similar expression patterns hinting at analogous gene functions, 1,682 genes induced in response to both Bgt- and Pst-induced stress were screened with a model-based clustering algorithm to identify distinct gene expression profiles among the identified DEGs and to choose a total of eight clusters, to maintain as few tight clusters as possible while including most of the distinct expression patterns (Figure 2). Each gray line in Figure 2 represents the expression pattern for an individual gene, and the single black line indicates the average behavior for induced genes in each cluster. Surprisingly, these overlapping genes showed different expression patterns and complex changes in response to Pst infection. This finding illustrated that these genes may perform different 
Table 3 Go characterization of overlapped DEGs responding to Pst and Bgt stress

\begin{tabular}{|c|c|c|c|c|c|}
\hline GO.ID & Term & Annotated & Significant & Expected & KS \\
\hline GO:0009405 & Pathogenesis & 221 & 4 & 6.7 & $2.30 \mathrm{E}-16$ \\
\hline GO:0036180 & Filamentous growth ... in response to biotic stimulus & 148 & 3 & 4.49 & $1.70 \mathrm{E}-13$ \\
\hline GO:0071216 & Cellular response to biotic stimulus & 235 & 5 & 7.13 & $6.40 \mathrm{E}-10$ \\
\hline GO:0035690 & Cellular response to drug & 278 & 6 & 8.43 & $1.60 \mathrm{E}-08$ \\
\hline GO:0006695 & Cholesterol biosynthetic process & 113 & 8 & 3.43 & 1.10E-07 \\
\hline GO:0055114 & Oxidation-reduction process & 5917 & 313 & 179.4 & $2.50 \mathrm{E}-07$ \\
\hline GO:0006805 & Xenobiotic metabolic process & 268 & 14 & 8.13 & 7.70E-06 \\
\hline GO:0006696 & Ergosterol biosynthetic process & 27 & 2 & 0.82 & 5.80E-05 \\
\hline GO:0010204 & Defense response signaling pathway, resistance gene-independent & 331 & 6 & 10.04 & $6.90 \mathrm{E}-05$ \\
\hline GO:0045471 & Response to ethanol & 147 & 4 & 4.46 & $9.00 \mathrm{E}-05$ \\
\hline GO:0030447 & Filamentous growth & 355 & 9 & 10.76 & 0.00014 \\
\hline GO:0046951 & Ketone body biosynthetic process & 16 & 2 & 0.49 & 0.00018 \\
\hline GO:0001914 & Regulation of T cell mediated cytotoxicity & 8 & 2 & 0.24 & 0.00021 \\
\hline GO:0034446 & Substrate adhesion-dependent cell spread... & 41 & 1 & 1.24 & 0.00021 \\
\hline GO:0001666 & Response to hypoxia & 637 & 34 & 19.31 & 0.00028 \\
\hline GO:0016045 & Detection of bacterium & 540 & 10 & 16.37 & 0.00029 \\
\hline GO:0042221 & Response to chemical stimulus & 17001 & 693 & 515.46 & 0.0003 \\
\hline GO:0006357 & Regulation of transcription from RNA pol... & 1097 & 19 & 33.26 & 0.00038 \\
\hline GO:0019265 & Glycine biosynthetic process, by transam... & 15 & 1 & 0.45 & 0.00051 \\
\hline GO:0044182 & Filamentous growth of a population of unicellular organisms & 272 & 8 & 8.25 & 0.00054 \\
\hline GO:0052548 & Regulation of endopeptidase activity & 325 & 8 & 9.85 & 0.00054 \\
\hline GO:0006890 & Retrograde vesicle-mediated transport, G... & 119 & 3 & 3.61 & 0.00055 \\
\hline GO:0009436 & Glyoxylate catabolic process & 13 & 1 & 0.39 & 0.0007 \\
\hline GO:0043603 & Cellular amide metabolic process & 466 & 19 & 14.13 & 0.00072 \\
\hline GO:0019252 & Starch biosynthetic process & 491 & 56 & 14.89 & 0.00076 \\
\hline GO:0010244 & Response to low fluence blue light stimu... & 20 & 1 & 0.61 & 0.0008 \\
\hline GO:0006144 & Purine nucleobase metabolic process & 109 & 5 & 3.3 & 0.00085 \\
\hline GO:0044281 & Small molecule metabolic process & 13733 & 583 & 416.38 & 0.00091 \\
\hline GO:0009628 & Response to abiotic stimulus & 12227 & 576 & 370.71 & 0.00095 \\
\hline GO:0051262 & Protein tetramerization & 246 & 15 & 7.46 & 0.00097 \\
\hline
\end{tabular}

functions in response to different stimuli, and implied that wheat triggered distinct genes and regulatory networks to antagonize Bgt and Pst infection.

Our results for the overlapping DEGs revealed changes in approximately $15 \%$ of the transcriptome, with most changes classified as stimulus-specific. This view illustrates the "fluid" nature of the transcriptome and the challenge faced in understanding the complexity of any given stress response.

\section{Key fungal defense-related genes and pathways}

Transcript profiling is capable of revealing pathways of gene expression involved in a defense response, therefore detailed gene-by-gene analysis should be informative because the temporary KEGG pathway and GO annotations were incomplete. A total of 10,583 DEGs were identified, and the range of DEG expression ratios was -235.33 to 187.78 for Pst stress-induced genes and -428.85 to 3944.00 for Bgt stress-induced genes. To identify the DEGs showing the greatest changes in transcript levels, the top $1 \%$ of up- and down-regulated genes in the Bgt and Pst treatments at each time point (listed in Additional file 1: Table S7) were analyzed.

Comparison of data for N9134 Pst- and Bgt-inoculated plants with mock-inoculated data revealed gene expression changes that included basal defense transcripts and transcripts specific to the establishment of a biotrophic interaction with fungi. Twelve of the 49 top differentially expressed transcripts significant for treatment were induced by Pst inoculation at $1 \mathrm{dpi}$, whereas 16 were repressed 

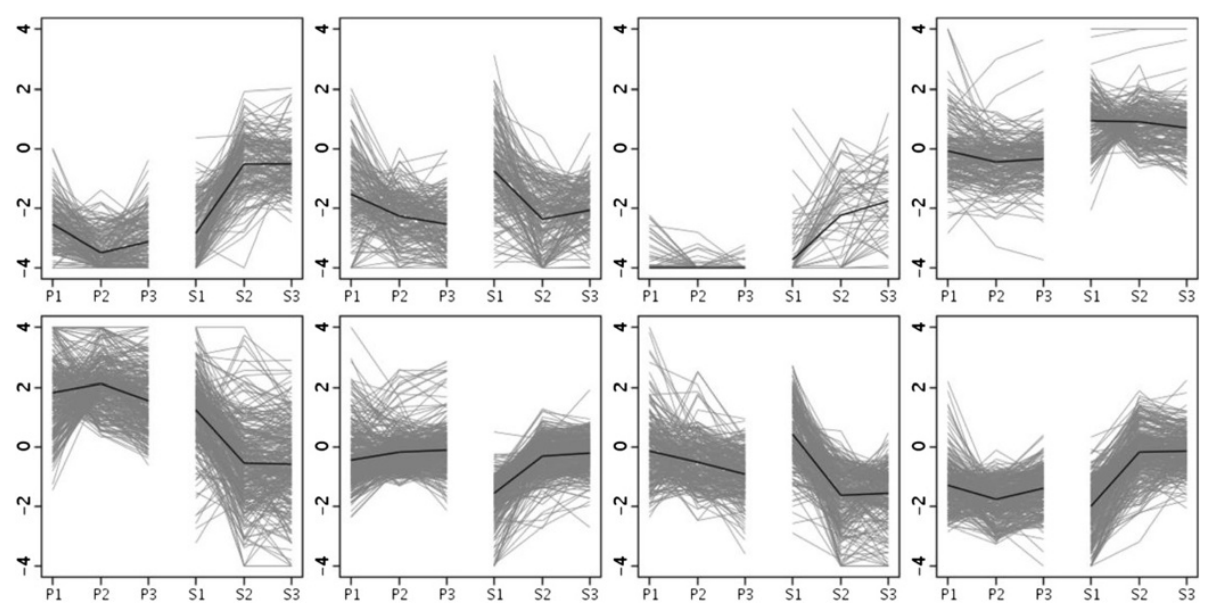

Figure 2 Clustering. The expression patterns of 1682 overlapped DE genes for 8 clusters. The horizontal axis indicates each time point and pathogen stress induction condition. The number 1, 2 and 3 mean that N9134 was infected at 1, 2 and 3 dpi, respectively. P represents powdery mildew E09 inoculation condition; S represents stripe rust pathogen CYR 31 inoculation. The vertical axis indicates the log2 fold change calculated between each condition and non-inoculation treatment. Each gray line symbolizes the expression pattern of one gene, and the bold back line illustrates the average expression pattern of all genes in each cluster under Bgt or Pst stress.

(Additional file 1: Table S7), 12 were down-regulated and three were induced by Pst infection at $2 \mathrm{dpi}$, while eight were repressed and five were up-regulated in response to Pst infection at $3 \mathrm{dpi}$. Ninety-five genes were ranked in the top $1 \%$ of genes dysregulated by Bgt inoculation, of which 35 were induced and four were repressed at $1 \mathrm{dpi}, 23$ were up-regulated and 26 were down-regulated at $2 \mathrm{dpi}$, and 17 were up-regulated and 35 were down-regulated at $3 \mathrm{dpi}$. As shown in Additional file 1: Table S7, defense-related transcripts accounted for $24.6 \%$ (33 out of 134) of the dysregulated transcripts, while $15(11.2 \%)$ were involved in signal transduction, 21 (15.7\%) in oxidation-reduction, 8 (6.0\%) in biological regulation, $9(6.7 \%)$ in metabolic processes, 5 (3.7\%) in protein/carbohydrate transport, 2 in development, 4 (3.0\%) in the tricarboxylic acid cycle, 1 in protein modification, and 34 (25.4\%) were of unknown/unclear function.

Comparing the top $1 \%$ transcriptomes after Pst and $B g t$ inoculation, most gene expression patterns showed opposing patterns in the two treatments (Additional file 1: Table S7), especially at the same time point. Those genes induced by $B g t$ infection were usually repressed in the same genotype by Pst infection, and vice versa. For example, this pattern was shown by L-type lectin-domain containing receptor kinase IX (T16.19639), hemoglobin 1 (T10.9286), luminal-binding protein (T10.42975), deltacadinene synthase isozyme XC14 (T13.45944), and hypothetical protein MTR_7g109740 (T4.16776).

\section{Plants sharing the microbe gene fragments antagonistic to fungal infection}

Blast searches of the $\mathrm{Nr}$ and Swissport databases showed that 89,672 annotated unigenes matched genes from
Aegilops tauschii (29.1\%), Triticum urartu (18.6\%), Hordeum vulgare (12.9\%), Brachypodium distachyon (4.7\%), Oryza sativa (4.4\%), and Marssonina brunnea (3.4\%), while 1565 unigenes (1.7\%) were related to T. aestivum in the current databases. Interestingly, 285 unigenes matched genes from Puccinia graminis (Pgt), of which 22 were expressed differentially in the Pst treatment, while 33 unigenes were similar to Apple stem pitting virus (ASPV) genes and 13 were induced in the $B g t$ treatment. A Blast search of the wheat genome with microbial sequences showed that some partial microbial genetic fragments were detected in the wheat genome. For example, a 115 bp fragment of T16_Unigene_BMK.81432 (homologous to arrestin domain-containing protein of $B g t$ ) was mapped to chromosomes 7B and 7D with identity of $100 \%$ and an $\mathrm{E}$ value of 9.0E-57, and a $227 \mathrm{bp}$ fragment of T16_Unigene_BMK.9260 (homologous to hypothetical protein PGTG_00959 of Pgt) was mapped to chromosome 1DL with an E value of 1.0E-103.

Ten pairs of gene-specific primers were designed based on de novo assembled suspected microbe sequences with the Primer 5.0 software and used in PCRs with genomic DNA extracted from non-infected wheat N9134 leaves as the template. The PCR products showed clear bands, and sequencing confirmed that the two fragments T16_ Unigene_BMK.9260 and T17_Unigene_BMK.8064, which were 400 and $280 \mathrm{bp}$ in length, respectively, were amplified from genomic DNA of the resistant wheat germplasm N9134 (Additional file 1: Figure S6). However, most of the checked unigenes could not be verified by sequencing of the PCR products amplified from the genomic DNA, although clear bands were observed in $1.5 \%$ agarose gels. 
This result indicated that most of the unigenes were transcribed from fungi, but some were homologous to wheat genes. Taken together, we inferred that the wheat line shared some genetic material with the microbes, which may be exploited by a virulent fungal pathogen to induce a response directed towards the wrong pathogen.

\section{High diversity of RGAs}

Because of the interest in wheat disease-resistance genes and plant-fungus interactions, the matched disease-resistance-related unigenes were clustered and those unigenes involved in plant-pathogen interaction KEGG pathways were compared between the Pst and Bgt treatments. In this transcriptome, we detected 2,064 unigenes that matched disease-resistance proteins with BlastX, including 374 unigenes with a NB-ARC conserved domain and 93 containing a NB-LRR domain. Seventyeight genes were regulated in response to Bgt infection of which 62 were induced, whereas 13 unigenes were disturbed by Pst inoculation of which only two were induced. Five regulated unigenes were shared by the two fungal stress responses and matched RPM1 and RGA4. This finding indicated that RGAs are highly diverse.

In addition, KEGG enrichment analysis showed that 40 differentially expressed unigenes were associated with plant-fungus interaction pathways and these genes dominated 20 crucial proteins or joints in the Bgt stress response, whereas nine differentially expressed unigenes that regulate seven points were detected in the Pst stress response. Comparison of the change in expression pattern of these enzymes at 1,2 , and 3 dpi indicated that 1 dpi was the most important time point because nearly all regulated genes were detected at this stage (Figure 3). For resistance to Pst infection, wheat manipulated flagellin-sensitive 2 (FLS2) and calcium-dependent protein kinase (CDPK) to trigger the HR response, and induced nonhost 1 (NHO1) at $1 \mathrm{dpi}$. The respiratory burst oxidase homolog $(\mathrm{RBOH}$, a plasma membrane NADPH oxidase) and heat shock protein (HSP90) were downregulated. However, the expression levels of FLS2 relative gene was severely decreased compared with that in noninoculated leaves, whereas $\mathrm{NHO} 1$ expression returned to normal at $2 \mathrm{dpi}$. Perhaps to compensate for the loss, the expression level of HSP90 was induced and attained a level similar to that of CDPK, whereas $\mathrm{RBOH}$ expression was restored to normal. At $3 \mathrm{dpi}$, FLS2, CDPK, and HSP90 genes maintained their respective expression levels observed at $2 \mathrm{dpi}$ at the cost of calmodulin/calmodulinlike (CaM/CML) repression.

In response to Bgt inoculation, wheat redeployed most pathways to trigger the $\mathrm{HR}$ response at $1 \mathrm{dpi}$, including PTI, ETI and NHO1. PTI was activated through the reactive oxygen species (ROS) and nitric oxide (NO) signaling pathway mediated by $\mathrm{CDPK}, \mathrm{RBOH}$ and $\mathrm{CaM} / \mathrm{CML}$,
FLS2, MKK1/2 (mitogen-activated protein kinase kinase), and WRKY25/33 involved a MAPK cascade. ETI was triggered via RIN4, RPS2, RAR1 (required for Mla12 resistance), SGT1 (suppressor of the G2 allele of SKP1), and HSP90. In addition, MIN7 (guanine nucleotide-exchange factor) and JAZ (jasmonate ZIM domain-containing protein) were induced to activate protein-mediated proteolysis and induce two chitin PRR proteins, Chitin elicitor receptor kinase 1 (CERK1) and Chitin elicitor-binding protein (CEBiP), but started to repress cyclic nucleotide-gated ion channel (CNGC) proteins at $1 \mathrm{dpi}$. However, most gene induction had ceased at 2 dpi except for six unigenes that matched CaM/CML, MKK1/2, HSP90, NHO1 and MIN7, and three enzymes were repressed, namely $\mathrm{RBOH}, \mathrm{JAZ}$ and MYC2 as CNGCs. NHO1 and MIN7 continued to be expressed, although the CaM/CML gene and Chitin PRR protein $\mathrm{CEBiP}$ were significantly down-regulated at $3 \mathrm{dpi}$.

Figure 3 shows that wheat accession N9134 employed multi-layered mechanisms to detect and combat pathogens, including preformed physical barriers and physiological and biochemical responses, upon recognition of pathogen-derived elicitors.

\section{Discussion}

In the present study, we performed triplicate deep transcriptome surveys in leaves of the same wheat line inoculated with Bgt and Pst. Using high-throughput RNA sequencing technology (RNA-Seq), we compared in detail the transcriptional differences and overlap between Bgt- and Pst-induced stress, and produced currently the most robust and reliable data for investigation of the response of wheat to fungal pathogen attack.

\section{Identification of differentially expressed genes and comparison with previously reported fungal transcriptome data}

Stripe rust and powdery mildew are among the most devastating diseases of wheat in cool regions. Several transcriptome and gene expression analyses of wheat following inoculation with these pathogens have been reported, such as the Yr5, Yr39 high-temperature, adult-plant resistance line versus Pst using the Affymetrix GeneChip Wheat Genome Array [15], 'Shaanmai 139' versus CYR 32 and 'Shuiyuan 11' against CYR 23 using suppression subtractive hybridization and cDNA-AFLP approaches [8]. Similarly, several reports on wheat-Bgt interaction have been published. For example, miRNAs and long non-coding RNA regulating the response to $B g t$ infection were analyzed using Solexa high-throughput sequencing [16,17], and gene expression profiling of wheat in response to Bgt infection [18]. Without the requirement for known gene sequences, high-throughput RNA sequencing provides more powerful data for observation of global gene expression profiles in different physiological processes in 


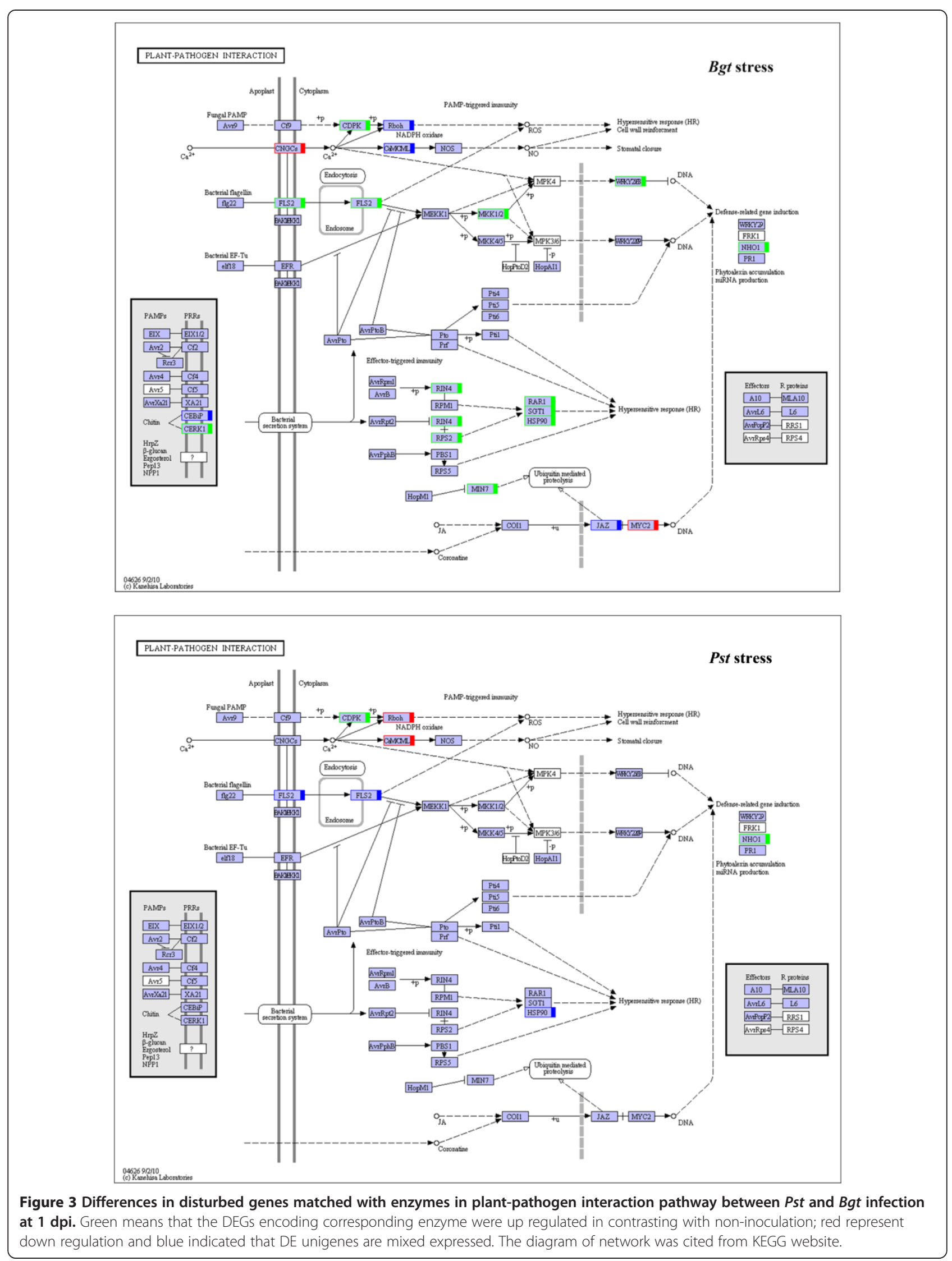


response to fungal attack than those contained in previous reports. Although we selected the top $1 \%$ dysregulated genes as representative, 134 unigenes were listed in Additional file 1: Table S6 owing to the thousands of genes observed in this study. The fungal-induced biological function genes could be divided into nine groups, which covered those reported in previous studies. Defense-related transcripts comprised the biggest group followed by oxidation-reduction, and signal transduction ranked as the third-largest group. The other groups consisted of metabolic, biological regulation, protein/carbohydrate transport, tricarboxylic acid cycle, development, and protein modification, as well as a tenth group categorized as "unknown/unclear". The results confirmed the expectation, based on observation of resistance-specific transcripts significantly induced at $1-3 \mathrm{dpi}$, that the top $1 \%$ genes were representative of the overall transcriptome. The mass sequence data obtained by deep sequencing of the wheat-fungus interactions provides a robust platform for future functional and molecular research than previously published data.

\section{Meta-analysis of the KEGG metabolism pathway}

In classical genetics, the pathogen-infected host plants and host responses usually showed monogenic control [19]. However, it is probable that thousands of genes can be detected in the early stages of infection, which causes induction of key resistance genes. This means that priming or core control genes must trigger other defenserelated and downstream genes. Accordingly, the analysis of pathway enrichment and metabolomics will open up the study of important host-pathogen systems, and may be helpful to identify the important factors that regulate such pathways.

In contrast with previous transcript analyses, we focused mainly on enriched pathways and biological processes in more detail, using meta-analysis of transcripts associated with race-specific pathogen resistance in addition to isolation of early response genes. When responding to pathogen attack, the host plant activates a network of pathways in an endeavor to impair pathogen invasion and to escape damage, which usually includes thousands of genes through a synergistic effect. In addition, bread wheat is hexaploid; as a consequence, characterization of multiple gene copies having mutual or compensatory interactions, such as those encoding the aforementioned disease resistance protein. In this study, we identified seven highly enriched KEGG pathways in response to Bgt infection and four in response to Pst infection, for example phenylalanine metabolism (Ko00360), phenylalanine, tyrosine and tryptophan biosynthesis (Ko00400), and carbon fixation in photosynthetic organisms (Ko00710). A meta-analysis is advantageous in helping to narrow the field of potential resistance gene analogues and in the search for the critical genes or triggers, and to construct an integrated metabolism network as shown in Figure 4. The final products of the Ko00360 and Ko00945 pathways (stilbenoid, diarylheptanoid and gingerol biosynthesis) are capsaicin and curcumin diglucoside. Capsaicin and curcumin have antibacterial and anticancer properties and reduce inflammatory reactions in mammals [20,21]; capsaicin also activates serine/ threonine kinase and AMP-activated kinase (AMPK), causing increased apoptosis [22]. This may imply that capsaicin and curcumin are similarly employed and processed as antifungal agents in plants, although further physiological and biochemical investigations are needed to test this hypothesis. In addition, this network of pathways will accelerate identification of upstream regulators and key transcription factors.

One component of this network is photosynthesis, and followed by photosynthesis-antenna proteins pathway, which has been reported to modulate plant defense responses induced by pathogen infection and by abiotic signals such as light, circadian rhythm, and temperature

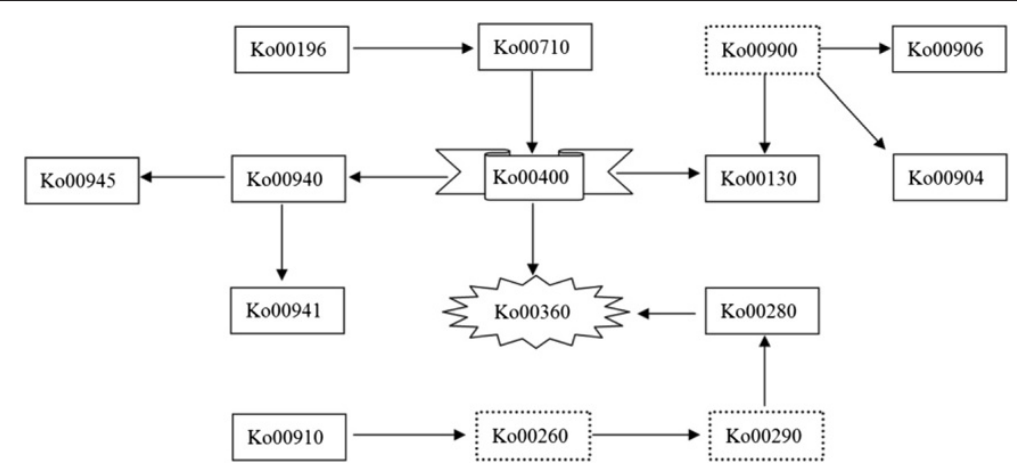

Figure 4 Overview of a hypothetical metabolism network model constructed with enriched DEGs KEGG pathways according to KEGG annotation. Aforementioned significant enriched KEE pathways were marked with solid frame, indistinctive KEGG pathways marked with dotted frame. Arrows indicated the fluid direction of metabolic products. 
[23]. In the present study, 12 enzymes that were dysregulated are participants in the light-harvesting chlorophyll protein complex (LHC) belonging to the photosynthesisantenna proteins pathway which was significantly enriched in both Bgt and Pst treatments. Modulation acts on specific key components of plant resistance, indicating that intricate integration of biotic and abiotic signals occurs.

\section{Relationship between transcriptome divergence and differences in resistance}

Although both Bgt and Pst are fungi, the number of genes induced by Bgt infection was 2.1 times higher than the number induced by Pst infection at $1 \mathrm{dpi}$, while the ratio was more than 5 times higher at 2 and $3 \mathrm{dpi}$. Genes that show highly correlated levels and temporal patterns of expression are often involved in similar functions or cellular processes [24]. Therefore, we clustered 1,682 overlapping DEGs to assess possible crosstalk between transcripts. Unexpectedly, those genes whose expression pattern was similar under Bgt attack showed differing temporal patterns of expression under Pst infection. This finding indicated that these genes have multiple or different functional roles in response to different biotic stresses. Considering that the wheat germplasm N9134 harbored two pathogen resistance loci, namely a stripe rust resistance gene on chromosome $1 \mathrm{~B}$ and a powdery mildew resistance gene on chromosome 5B [25], we hypothesized that this discrepancy results from different loci acting as a trigger or elicitor. Most importantly, this study has identified transcripts associated with multi-gene resistance in wheat N9134, which will be useful in future functional studies to identify relevant disease-responsive genes. This result also dispels the current belief that similar mechanisms are activated in response to $B g t$ and Pst infection.

\section{Microbe genes were shared with the wheat genome}

The ability to detect and mount a defense response against a potential pathogen has been paramount to the evolutionary and developmental success of plants. Apart from natural selection, an alternative model of evolution involving symbiotic relationships has been proposed and supported by experimental evidence $[19,26]$. Selection may act mutually and may drive parasite evolution and host-parasite co-evolution [27]. Likewise, the evolution of plants has also been shaped by molecular interactions with epiphytic, symbiotic, and pathogenic microbes [28]. RNA-RNA recombination is considered to be one of the strongest forces shaping the genomes of plant RNA viruses [29]. Profiting from gene sequences of rust fungi [30], the present transcriptome analysis found that the microbe genes or genomic hallmarks, including genes of $A S P V$ and Pgt origin, were shared by wheat and could be activated to respond to fungal attack. Based on present knowledge, a reasonable expectation is that wheat expresses the microbe-activated genes to outcompete or overcome hostile RNA as Huang suggested [31]. Paradoxically, molecular evidence proves that only partial unigenes are regulated by fungi, and the remarkable abundance of microbe-derivative genes in infected tissues cannot be an accurate reflection of resistance responses. A satisfactory explanation for the uneven spatial distribution of microbe genes in different fungus-infected tissues will require fine-tuned analyses taking into account the influence of fungal factors. Nevertheless, our results raise the possibility of genetic communication between plants and microbial pathogens, and further substantiates the previous hypothesis that recombination has occurred not only between viruses but even with host RNAs [29]. These features may represent tradeoff advantages of increased genetic variation independent of sexual recombination for biotrophy. Hence, it may provide a notable example of Dollo's law. This may explain why powdery mildews, stripe rust and possibly other biotrophic parasites became obligate pathogens.

\section{Key functional genes in plant-pathogen interaction}

Plants have evolved innate immune systems that recognize the presence of potential pathogens and initiate effective defense responses. Accumulation of resistance genes has been reported in the PTI and ETI pathways [4,32]. There are, however, some notable highlights or differences in resistance mechanisms against Pst and Bgt in wheat. First, previous observations indicated that CNGCs, which are members of the superfamily of ion channels with six transmembrane domains, are involved in diverse physiological functions and have an IQ domain [33,34], such as AtCNGC2 and AtCNGC4 responding to pathogen attack [35]. In the present study, the transcriptome analysis indicated that CNGC4 was repressed in wheat from 1 to 3 days after infection with the avirulent Bgt E09, whereas transcription of 19 genes similar to CNGC1, 5, 8, 14, 15, 17, and 20 were not disturbed in the wheat-pathogen interaction process. In addition, CaM/CML was markedly down-regulated after fungal inoculation, especially at 3 dpi. Although much evidence indicates that deregulation of $\mathrm{CaM} / \mathrm{CML}$ gene expression or loss of CaM/CML function strongly affects immune responses [36], this change in resistance phenotype was not observed in N9134. This finding indicated that bread wheat synthetically used multiple pathways to perceive and fight fungal infection in addition to the NOS signal pathway. Inspired by the function of DND2, a cyclic nucleotide-gated ion channel reported to accelerate cell death [35], we hypothesize that CNGC4 may play a similar role to resist attack by the powdery mildew pathogen because of the perfect 'no death phenotype' resistance of N9134 to Bgt E09.

Second, plant CDPKs are a key regulator of innate immune responses to pathogen-associated molecular pattern stimulation $[37,38]$, and a mutual activation circuit 
consisting of CPK5 and NADPH oxidase RBOH facilitates rapid signal propagation in the plant [39]. In the current study, we detected one CDPK gene in wheat that was positively persistent and four-fold regulated at all time points $(1,2$, and $3 \mathrm{dpi})$ in response to Pst attack, whereas three CDPKs were induced only at $1 \mathrm{dpi}$. Intriguingly, we found that the $\mathrm{RBOH}$ was selectively repressed or induced in the early stages of fungal attack, which provides a chance to identify in vivo substrates to unravel CDPK functions and correlate CDPKs with their corresponding substrates to establish their biological significance.

The leucine-rich repeat receptor kinases FLS2 contribute to resistance against bacterial infection in Arabidopsis thaliana [40]. The third notable difference in resistance mechanisms against Pst and Bgt infection in wheat was that the similar serine/threonine-protein kinase FLS2 genes were implicated in protecting wheat seedlings from infection by the two fungi. However, no flg22 or homolog was detected among the unigenes. Our results indicated that the elicitation of this basal immune response is an effective strategy for protecting the plant from both bacterial and fungal pathogens in wheat as well as Arabidopsis. We speculate that in wheat an alternative elicitor must interact with FLS2 to contribute to plant resistance.

\section{Conclusion}

The findings presented here provide evidence to clarify the hypothesis that wheat antagonizes stripe rust and powdery mildew infection with similar molecular mechanisms and gene transcripts because both pathogens are biotrophic fungi. In contrast, we found that a disease-resistant wheat line triggers various defense mechanisms to strengthen disease resistance, and that expression patterns of the same defense-associated genes were altered in adaptation to different pathogens. Transcripts of the infection-induced wheat genes were more abundant in Bgt-infected leaves than in Pst-infected leaves at an early stage following inoculation, especially of genes in plant-pathogen interaction pathways. Our experiment showed that the wheat line shared some microbial genetic materials with the pathogens, which may be exploited to resist infection by a virulent fungal pathogen. Our study provides new insights into the underlying mechanisms related to modulation and regulation of various biochemical pathways in response to fungal infection. Moreover, we provide a powerful platform for further exploration of disease-resistance genes, gene function, molecular research on wheat-fungus interactions, and marker development for classical genetics.

\section{Methods}

\section{Fungus and plant materials}

The winter wheat line N9134, developed at Northwest A\&F University, is a line that shows high resistance to Pst races CYR 29 and CYR 31 and is resistant to all Bgt races in China. This high level of resistance to Pst and Bgt is conferred by two all-stage resistance genes located on chromosomes $1 \mathrm{~B}$ and $5 \mathrm{BL}$, respectively. The Pst race CYR 31 was maintained by the College of Plant Protection of Northwest A\&F University. The Bgt isolate E09 was maintained on susceptible wheat 'Shaanyou 225'. The N9134 plants were cultivated in soil in a growth chamber at $18^{\circ} \mathrm{C}$ under a $16 \mathrm{~h}$ light/ $8 \mathrm{~h}$ dark photoperiod. Half of the 7day-old seedlings were inoculated with $B g t$ conidia from 'Shaanyou 225' seedlings infected 10 days previously. The other halves of the seedlings were inoculated with Pst race CYR 31. 'Shaanyou 225' and 'Huixianhong' were inoculated with E09 and CYR 31 to check that inoculation was successful or unsuccessful, respectively. The inoculated leaves of N9134 were harvested at $0,1,2$, and $3 \mathrm{dpi}$, frozen immediately in liquid nitrogen, and stored at $-80^{\circ} \mathrm{C}$. The test was carried out with three biological replications.

\section{EST library construction and sequencing}

Total RNA was extracted from samples of fungalinoculated leaves at the specified time points using the TRIzol reagent (BioFlux, Hang Zhou) method with a few modifications pertaining to DNase digestion and RNA purification. A small fraction of the RNA was electrophoresed in a $1 \%$ agarose gel to check its quality. Oligo(dT)-magnetic beads were used to enrich the mRNA, which was then broken into fragments with fragmentation buffer. First-strand cDNA was prepared using a reverse transcription-PCR system (Promega, Madison, WI, USA) with random hexamers. Second-strand cDNA was synthesized using RNase H, DNA polymerase I and dNTPs. Poly (A) and adaptor sequences were ligated to the ends of the repaired double-stranded cDNA after purification with a QiaQuick PCR kit. EST libraries were constructed by PCR amplification after checking the quality with agarose gel electrophoresis and sequenced with an Illumina HiSeq ${ }^{\mathrm{Tx}}$ 2000 platform by Biomarker Technology Co., Ltd (Beijing, China).

\section{Sequence processing and unigene library}

After sequencing, paired-end reads were checked and scored according to the CycleQ20 level standard (i.e., a base quality greater than 20 and an error probability of 0.01). After removinging low-quality reads, all reliable reads were assembled using the Trinity platform to reconstruct a unigene library for the wheat resistance line N9134 [41], and DEG analysis was performed with the bioconductor package DESeq [42]. Gene annotation and pathway identification were performed in accordance with the method described by Shi [43]. To organize genes into hierarchical categories and uncover gene regulatory networks on the basis of biological process, molecular function and cellular components, the DEGs were mapped 
to Gene Ontology (GO) terms and Kyoto Encyclopedia of Genes and Genomes (KEGG) pathways using the MAS molecular function annotation system (http://bioinfo.capitalbio. com/mas, 11 March 2012). GO terms and KEGG pathways with FDR-corrected $P$-values $<0.001$ were considered statistically significant. The RPKM values were used to examine the gene expression level distribution for each gene in each sample. In addition, the correlation coefficients $\left(R^{2}\right)$ between replicates were calculated.

\section{Quantitative real-time PCR analysis}

The SYBR Green Premix Ex Taq ${ }^{\mathrm{Tm}}$ II quantitative PCR system (Takara, Dalian) was used for qPCR analysis. All experiments involving q-PCR were performed on a 7300 Real-Time PCR System (Applied Biosystems, Foster City, CA, USA) using primers described in Additional file 1: Table S8. The RNA samples used as templates for RNASeq were the same as those used for qPCR. The tubulin gene was used as the internal control for normalization of qPCR data. Pearson correlation coefficients between the RNA-Seq and qPCR methods were calculated for eight selected genes across three time points under the stress treatment conditions, based on the average log2 fold change of three biological replicates. PCR was conducted according to the protocol described by Zhang et al. [44].

\section{Virus genes checked with wheat genome}

DNA extraction was carried out using the CTAB method from healthy leaves of wheat line N9134 seedlings. PCR amplifications were performed in total volume of $20 \mu \mathrm{l}$ following standard protocols. The amplification products were visualized on a $1.5 \%$ agarose gel. The microbe genespecific primers used in the PCRs were designed based on unigene sequences with Primer 5.0 software and synthesized by Beijing AuGCT DNA-SYN Biotechnology Co. (Beijing, China). The primers used are listed in Additional file 1: Table S8.

\section{Additional file}

Additional file 1: The sequence data from this study have been submitted to NCBI http://www.ncbi.nlm.nih.gov under accession No. PRJNA243835. Figure S1. Evaluate the reliability of RNA-Seq with qRT-PCR. Figure S2. MA scatter plot of gene expression level distributions for each treatment comparing to contrast. Figure $\mathbf{S 3}$. Volcano scatter plot of gene expression level distributions for Bgt treatment comparing to Pst. Figure S4a. Disturbedgenes matched with enzymes of ribosome pathway in Bgt infection. Figure S4b. Disturbedgenes matched with enzymes of ribosome pathway in Pst infection. Figure S5. Venn diagram to illustrate the number of DEGs shared by Pst and Bgt. Figure S6. PCR amplification verifies the fragments of microbe in gDNA of N9134. Table S1. Statistic correlation coefficients of biological replicates. Table S2. GO category (biological process) hits based on the algal functional annotation tool. Table S3. GO category (molecular function) hits based on the algal functional annotation tool. Table S4. GO category (cellular component) hits based on the algal functional annotation tool. Table S5. Significant KEGG enrichment pathway in responses to Pst and Bgt compared with each other. Table S6. KEGG pathway of stimulus-specific responses on Pst and Bgt compared with non-inoculation. Table S7. The detail of the top $1 \%$ up and down regulated DEGs in responding to fungi stress at each time points. Table S8. Primer sequences used in GRT-PCR with CDNA and PCR with gDNA.

\section{Competing interests}

The authors declare that they have no competing interests.

\section{Authors' contributions}

$\mathrm{HZ}$ and $\mathrm{WJ}$ designed the research. $\mathrm{HZ}$ and $\mathrm{YY}$ performed the research and contributed to the writing of the article. CW contributed to the development of material. HL, YF and YN contributed by collecting the samples and PCR and $\mathrm{PPCR}$ analysis. $\mathrm{HZ}$ and $\mathrm{ML}$ analyzed data. $\mathrm{YW}$ and $\mathrm{XL}$ contributed to writing. All authors read and approved the final manuscript.

\section{Acknowledgments}

This work was financially supported by National Key Basic Research Program of China (2013CB127700), National High-technology Research 863 Program of China (2011AA100103), and by the National Natural Science Foundation of China (31371612), as well as by the Fundamental Research Funds for the Central Universities (Northwest A\&F University, QN2011002). We would also like to thank Dr. Douglas Brown for critical reading of this manuscript.

Received: 18 July 2014 Accepted: 9 October 2014

Published: 15 October 2014

\section{References}

1. Kroupitski Y, Golberg D, Belausov E, Pinto R, Swartzberg D, Granot D, Sela S: Internalization of Salmonella enterica in leaves is induced by light and involves chemotaxis and penetration through open stomata. Appl Environ Microbiol 2009, 75:6076-6086.

2. Underwood W, Melotto M, He SY: Role of plant stomata in bacterial invasion. Cell Microbiol 2007, 9:1621-1629.

3. Jones JD, Dangl JL: The plant immune system. Nature 2006, 444:323-329.

4. Dodds PN, Rathjen JP: Plant immunity: towards an integrated view of plant-pathogen interactions. Nat Rev Genet 2010, 11:539-548.

5. Cantu D, Govindarajulu M, Kozik A, Wang M, Chen X, Kojima KK, Jurka J, Michelmore RW, Dubcovsky J: Next generation sequencing provides rapid access to the genome of Puccinia striiformis $\mathrm{f}$. sp. tritici, the causal agent of wheat stripe rust. PLOS One 2011, 6:e24230.

6. Coram TE, Settles ML, Chen X: Large-scale analysis of antisense transcription in wheat using the Affymetrix GeneChip Wheat Genome Array. BMC Genomics 2009, 10:253.

7. Hulbert SH, Bai J, Fellers JP, Pacheco MG, Bowden RL: Gene expression patterns in near isogenic lines for wheat rust resistance gene Lr34/Yr18. Phytopathology 2007, 97:1083-1093.

8. Wang X, Liu W, Chen X, Tang C, Dong Y, Ma J, Huang X, Wei G, Han Q, Huang L, Kang ZS: Differential gene expression in incompatible interaction between wheat and stripe rust fungus revealed by CDNA-AFLP and comparison to compatible interaction. BMC Plant Biol 2010, 10:9.

9. Fung RW, Gonzalo M, Fekete C, Kovacs LG, He Y, Marsh E, McIntyre LM, Schachtman DP, Qiu W: Powdery mildew induces defense-oriented reprogramming of the transcriptome in a susceptible but not in a resistant grapevine. Plant Physiol 2008, 146:236-249.

10. Eckey C, Korell M, Leib K, Biedenkopf D, Jansen C, Langen G, Kogel K: Identification of powdery mildew-induced barley genes by CDNA-AFLP: functional assessment of an early expressed MAP kinase. Plant Mol Biol 2004, 55:1-15.

11. Fauteux F, Chain F, Belzile F, Menzies JG, Belanger RR: The protective role of silicon in the Arabidopsis-powdery mildew pathosystem. Proc Natl Acad Sci U S A 2006, 103:17554-17559.

12. Zhao J, Zhang H-C, Yao J-N, Huang LL, Kang ZS: Confirmation of Berberis spp. as alternate hosts of Puccinia striiformis $\mathrm{f}$. sp. tritici on wheat in China. Mycosystema 2011, 30:895-900.

13. Benjamini $Y$, Hochberg $Y$ : Controlling the false discovery rate: a practical and powerful approach to multiple testing. J R Stat Soc Series B Stat Methodol 1995, 57(1):289-300. 
14. Schmid M, Davison TS, Henz SR, Pape UJ, Demar M, Vingron M, Scholkopf B, Weigel D, Lohmann JU: A gene expression map of Arabidopsis thaliana development. Nat Genet 2005, 37:501-506.

15. Coram TE, Settles ML, Chen X: Transcriptome analysis of high-temperature adult-plant resistance conditioned by Yr39 during the wheat-Puccinia striiformis f. sp. tritici interaction. Mol Plant Pathol 2008, 9:479-493.

16. Xin M, Wang Y, Yao Y, Song N, Hu Z, Qin D, Xie C, Peng H, Ni Z, Sun Q: Identification and characterization of wheat long non-protein coding RNAs responsive to powdery mildew infection and heat stress by using microarray analysis and SBS sequencing. BMC Plant Biol 2011, 11:61.

17. Xin M, Wang $Y$, Yao $Y$, Xie C, Peng H, Ni Z, Sun Q: Diverse set of microRNAs are responsive to powdery mildew infection and heat stress in wheat (Triticum aestivum L.). BMC Plant Biol 2010, 10:123.

18. Bhuiyan NH, Selvaraj G, Wei $Y$, King J: Gene expression profiling and silencing reveal that monolignol biosynthesis plays a critical role in penetration defence in wheat against powdery mildew invasion. $J$ Exp Bot 2009, 60:509-521.

19. Kang BC, Yeam I, Jahn MM: Genetics of plant virus resistance. Annu Rev Phytopathol 2005, 43:581-621.

20. Corson TW, Crews CM: Molecular understanding and modern application of traditional medicines: triumphs and trials. Cell 2007, 130:769-774.

21. Manjunatha $H$, Srinivasan $K$ : Protective effect of dietary curcumin and capsaicin on induced oxidation of low-density lipoprotein, iron-induced hepatotoxicity and carrageenan-induced inflammation in experimental rats. FEBS J 2006, 273:4528-4537.

22. Liu D, Zhu Z, Tepel M: The role of transient receptor potential channels in metabolic syndrome. Hypertens Res 2008, 31:1989-1995.

23. Hua J: Modulation of plant immunity by light, circadian rhythm, and temperature. Curr Opin Plant Biol 2013, 16:406-413.

24. Le Roch KG, Zhou Y, Blair PL, Grainger M, Moch JK, Haynes JD, De La Vega P, Holder AA, Batalov S, Carucci DJ, Winzeler EA: Discovery of gene function by expression profiling of the malaria parasite life cycle. Science 2003, 301:1503-1508.

25. Xue F, Ji W, Wang C, Zhang H, Yang B: High-density mapping and marker development for the powdery mildew resistance gene PmAS846 derived from wild emmer wheat (Triticum turgidum var. dicoccoides). Theor Appl Genet 2012, 124:1549-1560

26. Roossinck MJ: Symbiosis versus competition in plant virus evolution Nat Rev Microbiol 2005, 3:917-924.

27. Sacristán S, García-Arenal F: The evolution of virulence and pathogenicity in plant pathogen populations. Mol Plant Pathol 2008, 9:369-384.

28. Chisholm ST, Coaker G, Day B, Staskawicz BJ: Host-microbe interactions: shaping the evolution of the plant immune response. Cell 2006, 124:803-814.

29. Sztuba-Solinska J, Urbanowicz A, Figlerowicz M, Bujarski JJ: RNA-RNA recombination in plant virus replication and evolution. Annu Rev Phytopathol 2011, 49:415-443.

30. Duplessis S, Cuomo CA, Lin YC, Aerts A, Tisserant E, Veneault-Fourrey C, Joly DL, Hacquard S, Amselem J, Cantarel BL, Chiu R, Coutinho PM, Feaue N, Field M, Frey P, Gelhaye E, Goldberg J, Grabherr MG, Kodira CD, Kohler A Kües U, Lindquist EA, Lucas SM, Mago R, Mauceli E, Morin E, Murat C, Pangilinan $J$, Park R, Pearson $M$, et al: Obligate biotrophy features unraveled by the genomic analysis of rust fungi. Proc Natl Acad Sci U S A 2011, 108:9166-9171.

31. Huang XL, Chen XM, Coram T, Wang MN, Kang ZS: Gene expression profiling of Puccinia striiformis $\mathrm{f}$. sp. tritici during development reveals a highly dynamic transcriptome. J Genet Genomics 2011, 38:357-371.

32. Schwessinger $B$, Ronald PC: Plant innate immunity: perception of conserved microbial signatures. Annu Rev Plant Biol 2012, 63:451-482.

33. Fischer C, Kugler A, Hoth S, Dietrich P: An IQ domain mediates the interaction with calmodulin in a plant cyclic nucleotide-gated channel. Plant Cell Physiol 2013, 54:573-584.

34. Urquhart W, Chin K, Ung H, Moeder W, Yoshioka K: The cyclic nucleotidegated channels AtCNGC11 and 12 are involved in multiple $\mathrm{Ca}^{2+}$-dependent physiological responses and act in a synergistic manner. J Exp Bot 2011, 62:3671-3682.

35. Jurkowski Gl, Smith RK Jr, Yu IC, Ham JH, Sharma SB, Klessig DF, Fengler KA, Bent AF: Arabidopsis DND2, a second cyclic nucleotide-gated ion channel gene for which mutation causes the "defense, no death" phenotype. Mol Plant Microbe Interact 2004, 17:511-520

36. Cheval C, Aldon D, Galaud JP, Ranty B: Calcium/calmodulin-mediated regulation of plant immunity. Biochim Biophys Acta 1833, 2013:1766-1771.
37. Boudsocq M, Sheen J: CDPKs in immune and stress signaling. Trends Plant Sci 2013, 18:30-40.

38. Coca M, San Segundo B: AtCPK1 calcium-dependent protein kinase mediates pathogen resistance in Arabidopsis. Plant J 2010, 63(3):526-540.

39. Dubiella U, Seybold H, Durian G, Komander E, Lassig R, Witte CP, Schulze WX Romeis T: Calcium-dependent protein kinase/NADPH oxidase activation circuit is required for rapid defense signal propagation. Proc Natl Acad Sci U S A 2013, 110:8744-8749.

40. Gomez-Gomez L, Boller T: FLS2: an LRR receptor-like kinase involved in the perception of the bacterial elicitor flagellin in Arabidopsis. Mol Cell 2000, 5:1003-1011.

41. Haas BJ, Papanicolaou A, Yassour M, Grabherr M, Blood PD, Bowden J, Couger MB, Eccles D, Li B, Lieber M, MacManes MD, Ott M, Orvis J, Pochet N, Strozzi F, Weeks N, Westerman R, William T, Dewey CN, Henschel R, LeDuc RD, Friedman N, Regev A: De novo transcript sequence reconstruction from RNA-seq using the Trinity platform for reference generation and analysis. Nat Protoc 2013, 8:1494-1512.

42. Anders $S$, Huber W: Differential expression analysis for sequence count data. Genome Biol 2010, 11:R106.

43. Shi YH, Zhu SW, Mao XZ, Feng JX, Qin YM, Zhang L, Cheng J, Wei LP, Wang ZY, Zhu YX: Transcriptome profiling, molecular biological, and physiological studies reveal a major role for ethylene in cotton fiber cell elongation. Plant Cell 2006, 18:651-664.

44. Zhang H, Hu YG, Yang BJ, Xue F, Wang CY, Kang ZS, Ji WQ: Isolation and characterization of a wheat IF2 homologue required for innate immunity to stripe rust. Plant Cell Rep 2013, 32:591-600.

doi:10.1186/1471-2164-15-898

Cite this article as: Zhang et al.: Large-scale transcriptome comparison reveals distinct gene activations in wheat responding to stripe rust and powdery mildew. BMC Genomics 2014 15:898.

\section{Submit your next manuscript to BioMed Central and take full advantage of:}

- Convenient online submission

- Thorough peer review

- No space constraints or color figure charges

- Immediate publication on acceptance

- Inclusion in PubMed, CAS, Scopus and Google Scholar

- Research which is freely available for redistribution

Submit your manuscript at www.biomedcentral.com/submit
C Biomed Central 